\title{
@creative
}

ISSN 2590-9770

The Art of Discrete and Applied Mathematics 4 (2021) \#P1.02

https://doi.org/10.26493/2590-9770.1304.2cf

(Also available at http://adam-journal.eu)

\section{A note on a candy sharing game*}

\author{
Deepak Bal \\ Department of Mathematics, Montclair State University, Montclair, NJ, USA \\ Joseph DeGaetani \\ Department of Mathematical Sciences, University of Delaware, Newark, DE, USA
}

Received 9 July 2019, accepted 3 February 2020, published online 10 September 2020

\begin{abstract}
Suppose $k$ students sit in a circle and are each distributed some initial amount of candy. Each student begins with an even amount of candy, but their individual amounts may vary. Upon the teacher's signal, each student passes half of their candy to their left and keeps half. After this step, any student with an odd amount of candy receives an extra piece. The game ends if all the students are holding the same amount of candy. We prove, in a generalized setting, that for any initial distribution of $n$ pieces of candy, the game terminates after $O(\log n)$ many iterations and each student ends with $\frac{n}{k}+O(\log n)$ many pieces. Moreover, there exist initial distributions for which the $O(\log n)$ term cannot be improved.
\end{abstract}

Keywords: Games on graphs, Markov chains.

Math. Subj. Class. (2020): 05C20, 60J10

\section{Introduction}

In this note, we analyze a game referred to as the candy sharing game. Suppose a fixed number $k$ many students sit in a circle and are each distributed some initial amount of candy. Each student begins with an even amount of candy, but their individual amounts may vary. Upon the teacher's signal, each student passes half of their candy to their left and keeps half of their candy. After this step, any student with an odd amount of candy receives an extra piece. The game ends if all the students are holding the same amount of candy. Does the game end after finitely many steps for any initial distribution?

This question originated as a problem in the Beijing Math Olympiad [2]. A web search shows it remains popular as a fun activity for budding mathematicians as well as a challenge

\footnotetext{
${ }^{*}$ The authors would like to thank the referees for their careful reading and numerous suggestions which helped improve the presentation of the paper. Also, for pointing out an error in an earlier version.

E-mail addresses: deepak.bal@montclair.edu (Deepak Bal), degaetanij2@montclair.edu (Joseph DeGaetani)
} 
in computer coding competitions. The answer to the question is "yes" and it boils down to the following three observations: (1) The maximum amount of candy held by any player can never increase. (2) The number of players holding the currently minimum amount of candy will decrease by at least one each step. Thus the minimum amount will increase after at most $k$ steps. (3) Once the maximum and minimum are equal, the game terminates.

In [4], Iba and Tanton consider a generalized version of the game in which each player fixes an integer and at each step shares portions of their candy to some subset of the other players. After receipt of candy from the other players, they round up to the nearest multiple of their chosen integer. They prove that under certain conditions, such games are also bounded, i.e., reach a stable state after some finite number of moves. In [1], Cairns considers a version of the game where at each step, each player with more than one piece of candy passes one piece to their left and one to their right. The game is played until it settles into a fixed state or an oscillatory pattern. He completely characterizes the long term behavior given any initial distribution in the case then the number of students and candies are both $k$.

For the original candy sharing game, given an initial distribution of candy, predicting the length of the game and the final stabilizing amount is an intriguing open question. The main purpose of this note is to provide an upper bound on each of the above parameters which is tight infinitely often (at least in the case when the number of players $k=3$ ).

We will consider the candy sharing game played in a generalized setting. We say a directed graph $G$ is $d$-regular if every vertex has in- and out- degree equal to $d$. The candy sharing game can be played on any $d$-regular graph $G$ as follows. Each vertex (player) is distributed some amount of candy which is divisible by $d$. At each step, each player hands $1 / d$ proportion of their candy to each of their out-neighbors and is handed candy from each of their in-neighbors. After this, each student is handed $0,1, \ldots$, or $d-1$ pieces of candy to ensure they are holding a multiple of $d$. Thus the original candy sharing game is one played on the directed cycle of length $k$ with a directed loop at each vertex. A directed graph $G$ is strongly connected if between any two vertices $u$ and $v$, there exists a directed $u, v$-path. $G$ is aperiodic if the greatest common divisor of the lengths of its cycles is 1 . Our main theorem is as follows.

Theorem 1.1. Let $k \geq d \geq 2$ be fixed. For any $\boldsymbol{d}=\left(a_{1}, \ldots, a_{k}\right)$ such that $\sum_{i=1}^{k} a_{i}=n$ the candy sharing game, played with initial candy distribution $\boldsymbol{d}$, on a strongly connected, aperiodic, d-regular directed graph $G$ ends in $O(\log n)$ turns, and every player will be holding $\frac{n}{k}+O(\log n)$ pieces of candy.

The next proposition shows that, at least in some specific cases, the order of the $\log n$ term in the final distribution cannot be improved.

Proposition 1.2. There exist infinitely many values of $n$ such that the candy sharing game with initial distribution $\boldsymbol{d}=(n, 0,0)$ played on a directed cycle of length 3 with a directed loop on each vertex terminates with after $\Omega(\log n)$ turns with every player holding $\frac{n}{3}+$ $\Omega(\log n)$ pieces of candy.

In the next section we introduce the definitions and results from the theory of Markov chains necessary for the proof. In Section 3 we prove Theorem 1.1. 


\section{Notation and background}

Throughout the paper, we consider $k$ and $d$ to be fixed, so $O(\cdot)$ and $\Omega(\cdot)$ notation is suppressing constants which may depend on $k$ and $d$. All logarithms are natural unless otherwise stated. Vectors will be denoted by boldface characters and for a vector $\mathbf{v}$, we use the notation $\mathbf{v}(i)$ to denote the $i$ th entry of $\mathbf{v}$. Suppose the game is played on a directed graph $G$ with vertex set $V(G)=\{1, \ldots, k\}$ and edge set $E(G)$. For $t=0,1,2, \ldots$, let $\mathbf{d}_{t}=\left(a_{1, t}, a_{2, t}, \ldots, a_{k, t}\right)$ where $a_{i, t}$ represents the amount of candy held by player $i$ after $t$ steps of the game. One can check that for any $t \geq 1$, if $\mathbf{d}_{t-1}=\left(b_{1}, \ldots, b_{k}\right)$, then we have the $i$ th entry of $\mathbf{d}_{t}$ is given by

$$
\mathbf{d}_{t}(i)=d \cdot\left\lceil\frac{1}{d} \sum_{j: j i \in E(G)} \frac{b_{j}}{d}\right\rceil .
$$

At each step of the game, if a player has an amount of candy not divisible by $d$, then they receive extra pieces. Each piece of candy introduced in this way is referred to as a draw. Note that if the game terminates with each player holding $s$ pieces of candy, then the total amount of candy at termination is $s k$. So the total number of draws in a candy game with $n$ pieces initially distributed is $s k-n$. The number of draws up to and including turn $t$ is denoted $\Delta_{t}$.

To continue we need some definitions and results regarding Markov chains. All notation and definitions follow those in [5]. A Markov chain with state space $\Omega$ and transition matrix $P$ is a sequence of random variables $\left(X_{0}, X_{1}, \ldots\right)$ on $\Omega$ such that for all $i \geq 0$, if $X_{i}$ has distribution $\mu$, then $X_{i+1}$ has distribution $\mu P$. We represent distributions on $\Omega$ as row vectors and $P$ as an $|\Omega| \times|\Omega|$ matrix where entry $P_{i j}$ represents the probability of transitioning from state $i$ to state $j$.

We say a chain is irreducible if, for any two states, there is a finite number of steps in which it is possible to transition from one state to the other with positive probability. This number of steps may be dependent on the chosen states. A chain is aperiodic if, for each state, the greatest common divisor of the set of times that it is possible to transition from the state back to itself is 1 .

Further, a distribution $\pi$ is stationary for $P$ if $\pi P=\pi$. Any irreducible chain has a unique stationary distribution, and each starting state will converge to the stationary. The total variation distance between two probability distributions, $\mu$ and $\nu$ is given by $\|\mu-\nu\|_{T V}=\max _{A \subset \Omega}|\mu(A)-\nu(A)|$. There is a convenient formula for calculating the total variation distance, given by

$$
\|\mu-\nu\|_{T V}=\frac{1}{2} \sum_{x \in \Omega}|\mu(x)-\nu(x)| .
$$

As we are concerned with how quickly the game stabilizes, we will make use of the following theorem (Thm 4.9 in [5]) which gives a bound on the rate of convergence of an irreducible aperiodic Markov chain.

Theorem 2.1 (Convergence Theorem). If $P$ is the transition matrix of an irreducible and aperiodic chain, with stationary distribution $\pi$, then for any initial probability distribution $x$ on state space $\Omega$, there exist constants $\alpha \in(0,1)$ and $C>0$ such that for all $t \geq 0$, $\left\|x P^{t}-\pi\right\|_{T V} \leq C \alpha^{t}$. 


\section{Proof of Theorem 1.1}

Proof of Theorem 1.1. Given a $d$-regular directed graph $G$ on vertex set $\{1,2, \ldots, k\}$, we may form a $k \times k$ transition matrix $P$ whose $i j$ entry is $1 / d$ if $i j$ is an edge, and 0 otherwise. We can view the Markov chain with transition matrix $P$ as a randomized candy sharing game where at each stage, vertices no longer draw, but instead individually distribute each piece of candy uniformly at random to one of its out-neighbors. As an example, the transition matrix for the original candy sharing game is given by

$$
\left.P=\left[\begin{array}{cccccc}
1 / 2 & 1 / 2 & 0 & 0 & \ldots & 0 \\
0 & 1 / 2 & 1 / 2 & 0 & \ldots & 0 \\
0 & 0 & 1 / 2 & 1 / 2 & \ldots & 0 \\
\vdots & \vdots & \vdots & \vdots & \ddots & \vdots \\
1 / 2 & 0 & 0 & 0 & \ldots & 1 / 2
\end{array}\right]\right\} k .
$$

Let $\mathbf{d}_{0}=\left(a_{1}, \ldots, a_{k}\right)$ with $n=\sum a_{i}, \tilde{\mathbf{c}}_{0}=\mathbf{d}_{0}$, and for $t \geq 1$, let $\tilde{\mathbf{c}}_{t}=\tilde{\mathbf{c}}_{t-1} P=\tilde{\mathbf{c}}_{0} P^{t}$. Then $\tilde{\mathbf{c}}_{t}(i)$ represents the expected amount of candy held by vertex $i$ after $t$ turns of the randomized candy sharing game. Let $\mathbf{c}_{t}=\frac{1}{n} \tilde{\mathbf{c}}_{t}$.

Note that since the randomized candy sharing game has no draws, we have the following inequalities:

$$
\begin{aligned}
& \min \left(\mathbf{d}_{t}\right) \geq \min \left(\tilde{\mathbf{c}}_{t}\right) \\
& \max \left(\mathbf{d}_{t}\right) \leq \max \left(\tilde{\mathbf{c}}_{t}\right)+\Delta_{t} .
\end{aligned}
$$

Lemma 3.1. If a candy sharing game is played on a strongly connected, aperiodic, $d$ regular directed graph $G$ with $k$ vertices, then in at most $k^{2}-2 k+2$ turns either the game will terminate or the minimum amount of candy held by any player will increase.

Proof. Let $t$ represent the current turn of the game, with $t=0$ being the initial distribution. Let $\tilde{\mathbf{c}}_{t}=\mathbf{d}_{t}=\left(a_{1}, a_{2}, \ldots, a_{k}\right)$ with $a_{i} \in \mathbb{Z}_{+}$for $i=1, \ldots k$, and further assume that not all entries of this vector are equal (meaning the game has not terminated). In particular, some entry of $\tilde{\mathbf{c}}_{t}$ is larger than $\min \left(\tilde{\mathbf{c}}_{t}\right)$. Given that $G$ is $d$-regular the transition matrix, $P$, for this chain is doubly stochastic, that is, all of its row and column sums are 1. It is easily verifiable that the product of doubly stochastic matrices is also doubly stochastic.

A theorem of Wielandt (see [6]) says that for a $k \times k$, irreducible, non-negative matrix $P$, there exists an integer $r \leq k^{2}-2 k+2$ such that $P_{i j}^{r}>0$ for all $i, j$. (In fact, a theorem of Dulmage and Mendelsohn [3] says that if $P$ additionally has a non-zero diagonal entry, we have $P^{r}$ has all positive entries for some $r \leq 2 k-2$.)

Advancing the randomized game, $\tilde{\mathbf{c}}$, by $r$ turns and letting $A_{1}, \ldots A_{k}$ represent the columns of matrix $P^{r}$, we have

$$
\tilde{\mathbf{c}}_{t+r}=\tilde{\mathbf{c}}_{t} P^{r}=\left(\tilde{\mathbf{c}}_{t} \cdot A_{1}, \tilde{\mathbf{c}}_{t} \cdot A_{2}, \ldots, \tilde{\mathbf{c}}_{t} \cdot A_{k}\right) .
$$

Since $P^{r}$ is doubly stochastic, each entry of $\tilde{\mathbf{c}}_{t+r}$ is a weighted average of the entries of $\tilde{\mathbf{c}}_{t}$. Since there exist entries of $\tilde{\mathbf{c}}_{t}$ that are larger than $\min \left(\tilde{\mathbf{c}}_{t}\right)$, each weighted average is larger than $\min \left(\tilde{\mathbf{c}}_{t}\right)$. Thus, using (3.1), we have

$$
\min \left(\mathbf{d}_{t+r}\right) \geq \min \left(\tilde{\mathbf{c}}_{t+r}\right)>\min \left(\tilde{\mathbf{c}}_{t}\right)=\min \left(\mathbf{d}_{t}\right) .
$$


We can clearly see that if $\max \left(\mathbf{d}_{t}\right)-\min \left(\mathbf{d}_{t}\right)<1$ for some $t$, then the discrete game has ended. Let $\boldsymbol{\pi}=\left(\frac{1}{k}, \frac{1}{k}, \ldots, \frac{1}{k}\right)$. Then one can check that $\boldsymbol{\pi} P=\boldsymbol{\pi}$, that is, $\boldsymbol{\pi}$ is the stationary distribution for $P$. Let $\alpha$ and $C$ be given by Theorem 2.1 for matrix $P$ and initial distribution $\mathbf{c}_{0}$. Then after $t$ steps we have $\left\|\mathbf{c}_{t}-\boldsymbol{\pi}\right\|_{T V}<C \alpha^{t}$. Let $t_{0}=\left\lceil\frac{\log (2 C n)}{\log \left(\frac{1}{\alpha}\right)}\right\rceil$. Then

$$
\left\|\mathbf{c}_{t_{0}}-\boldsymbol{\pi}\right\|_{T V}<\frac{1}{2 n}
$$

Using the inequalities from (3.1), followed by normalizing the randomized game and utilizing the triangle inequality we have

$$
\begin{aligned}
\left|\max \left(\mathbf{d}_{t}\right)-\min \left(\mathbf{d}_{t}\right)\right| & \leq\left|\max \left(\tilde{\mathbf{c}}_{t}\right)-\min \left(\tilde{\mathbf{c}}_{t}\right)\right|+\Delta_{t} \\
& \leq n\left|\max \left(\mathbf{c}_{t}\right)-\min \left(\mathbf{c}_{t}\right)\right|+\Delta_{t} \\
& \leq n\left(\left|\max \left(\mathbf{c}_{t}\right)-\frac{1}{k}\right|+\left|\min \left(\mathbf{c}_{t}\right)-\frac{1}{k}\right|\right)+\Delta_{t} .
\end{aligned}
$$

In the above sum, we compare the distance between two entries of $\mathbf{c}_{t}$ and two entries of $\pi$. Including the remaining distances leads to the next inequality as $k \geq 2$.

$$
n\left(\left|\max \left(\mathbf{c}_{t}\right)-\frac{1}{k}\right|+\left|\min \left(\mathbf{c}_{t}\right)-\frac{1}{k}\right|\right)+\Delta_{t} \leq n \sum_{i=1}^{k}\left|\mathbf{c}_{t}(i)-\frac{1}{k}\right|+\Delta_{t} .
$$

By (2.1), we have

$$
n \sum_{i=1}^{k}\left|\mathbf{c}_{t}(i)-\frac{1}{k}\right|+\Delta_{t}=2 n\left\|\mathbf{c}_{t}-\boldsymbol{\pi}\right\|_{T V}+\Delta_{t}
$$

Now, $\Delta_{t}$ is bounded above by $(d-1) k t$, since at most every player will have to draw $(d-1)$ pieces every turn. Therefore, after $t_{0}$ turns, we have

$$
\begin{aligned}
\left|\max \left(\mathbf{d}_{t_{0}}\right)-\min \left(\mathbf{d}_{t_{0}}\right)\right| & \leq 2 n\left\|\mathbf{c}_{t_{0}}-\boldsymbol{\pi}\right\|_{T V}+\Delta_{t_{0}} \\
& \leq 2 n\left(\frac{1}{2 n}\right)+(d-1) k t_{0} \\
& =1+(d-1) k \cdot \frac{\log (2 C n)}{\log \left(\frac{1}{\alpha}\right)}
\end{aligned}
$$

where in the second inequality we have used (3.2). Thus we have that there exists a constant $C^{\prime}=C^{\prime}(k, d)$ such that after $t_{0}$ turns, $\left|\max \left(\mathbf{d}_{t_{0}}\right)-\min \left(\mathbf{d}_{t_{0}}\right)\right|<C^{\prime} \log n$.

Recall that $\max \left(\mathbf{d}_{t}\right)$ cannot increase and by Lemma 3.1, $\min \left(\mathbf{d}_{t}\right)$ is guaranteed to increase every $k^{2}-2 k+2 \leq k^{2}$ turns. Therefore, after at most $k^{2} C^{\prime} \log n$ more turns, $\left|\max \left(\mathbf{d}_{t}\right)-\min \left(\mathbf{d}_{t}\right)\right|$ will be less than 1 , and thus the game will have ended. From this we know the total number of turns the game took is at most $t_{0}+k^{2} C^{\prime} \log n=\frac{\log (2 C n)}{\log \left(\frac{1}{\alpha}\right)}+$ $k^{2} C^{\prime} \log n<C^{\prime \prime} \log n$ for some constant $C^{\prime \prime}=C^{\prime \prime}(k, d)$. At worst, each player draws $(d-1)$ pieces of candy every turn. So the total amount of candy at the end of the game is at most $n+(d-1) k C^{\prime \prime} \log n$ implying that each player has $\frac{n}{k}$ plus at most $O(\log n)$ pieces. 


\section{Proof of Proposition 1.2}

Proof of Proposition 1.2. Consider the sequence $\left(r_{i}\right)_{i=1}^{\infty}$ defined recursively by $r_{\ell}=4 r_{\ell-1}+2$ and $r_{1}=2$. We examine the game played on a directed cycle of length 3 with loops at each vertex. With initial distribution $\mathbf{d}=\left(r_{\ell}, 0,0\right)$, the sequence of states is as follows. An arrow indicates advancing a turn, and a number over the arrow represents how many pieces were drawn that turn. For $\ell \geq 2$,

$$
\begin{aligned}
\left(r_{\ell}, 0,0\right)=\left(4 r_{\ell-1}+2,0,0\right) & \stackrel{+2}{\longrightarrow}\left(2 r_{\ell-1}+2,2 r_{\ell-1}+2,0\right) \\
& \stackrel{+2}{\longrightarrow}\left(r_{\ell-1}+2,2 r_{\ell-1}+2, r_{\ell-1}+2\right) .
\end{aligned}
$$

Notice that each player is holding at least $r_{\ell-1}+2$ pieces of candy. We can imagine that they each divide their current piles into two: an inner pile consisting of the $r_{\ell-1}+2$ pieces, and an outer pile containing the remainder. The players would then continue by playing two concurrent games, following the game procedure on the inner pile and outer pile simultaneously. Since the inner pile is the same amount for each player, that game has already terminated and will no longer draw extra pieces. The only draws will then come from the outer game which, after invoking symmetry, is equivalent to the game played with initial distribution $\mathbf{d}^{\prime}=\left(r_{\ell-1}, 0,0\right)$.

Further, note that the $r_{\ell}$ sequence has binary representation

$$
10,1010,101010,10101010, \ldots
$$

where the $\ell^{t h}$ element is the digits (10) repeated $\ell$ times. Each time the above recursion is applied to a game of the form $\left(r_{\ell}, 0,0\right)$, two turns elapse, four pieces of candy are drawn and two digits are removed from the binary representation of the initial candy amount. We finally see that we need to draw 4 times the length of a base 2 expansion of $r_{\ell}$, which is logarithmic. Therefore, letting $n=r_{\ell}$, the sequence of games played with initial distribution $\mathbf{d}=(n, 0,0)$ will terminate after $\Theta(\log n)$ turns with each player holding $\frac{n}{3}+\Theta(\log n)$ many pieces of candy.

\section{Conclusion}

In this note, we have proved a relationship between the candy sharing game with rounding and a Markov chain without rounding and used the convergence theorem to find a bound on the length of the candy sharing game. The main open problem in candy sharing is to find a closed form expression for the number of rounds and ending amount of candy in terms of the initial candy distribution. It seems this may be quite difficult. Another interesting problem may be to try and prove a result similar to ours for the general games considered in [4].

\section{ORCID iDs}

Deepak Bal (D) https://orcid.org/0000-0003-1441-1823

\section{References}

[1] G. Cairns, Equitable candy sharing, Amer. Math. Monthly 124 (2017), 518-526, doi:10.4169/ amer.math.monthly.124.6.518. 
[2] G. Chang and T. W. Sederberg, Over and over again, volume 39 of New Mathematical Library, Mathematical Association of America, Washington, DC, 1997.

[3] A. L. Dulmage and N. S. Mendelsohn, Gaps in the exponent set of primitive matrices, Illinois J. Math. 8 (1964), 642-656, http: / / projecteuclid.org/euclid.ijm/1256059464.

[4] G. Iba and J. Tanton, Candy sharing, Amer. Math. Monthly 110 (2003), 25-35, doi:10.2307/ 3072341.

[5] D. A. Levin, Y. Peres and E. L. Wilmer, Markov chains and mixing times, American Mathematical Society, Providence, RI, 2009, with a chapter by James G. Propp and David B. Wilson.

[6] H. Schneider, Wielandt's proof of the exponent inequality for primitive nonnegative matrices, Linear Algebra Appl. 353 (2002), 5-10, doi:10.1016/S0024-3795(02)00414-7. 